\title{
Influência da mídia no consumo de alimentos ultraprocessados e no estado nutricional de escolares
}

\author{
Media's influence over consumption of ultra-processed foods and interference of \\ nutritional status of children students
}

La Influencia de los medios de comunicación sobre el consumo de alimentos ultraprocesados e su interferencia del estado nutricional de los niños estudiantes

Janete da Costa Barbosa de Melo ${ }^{1 *}$, Gabriella Freire Lustoza', Daniela Fortes Neves Ibiapina', Liejy Agnes Raposo dos Santos Landim¹.

\section{RESUMO}

Objetivo: Analisar a influência da mídia no consumo de ultraprocessados por escolares e sua relação com o estado nutricional. Métodos: Estudo descritivo, de abordagem transversal. Coletaram-se dados antropométricos, consumo de alimentos ultraprocessados e uso de aparelhos eletrônicos. A pesquisa foi realizada em uma escola da rede privada no município de Teresina-PI. Foram selecionados 75 escolares, dos turnos manhã e tarde, de ambos os sexos, com idade de 7 a 10 anos. Resultados: Observou-se uma maior prevalência de excesso de peso, sendo 22,7\% (17) de sobrepeso, $14,7 \%$ (11) obesidade e 10,7\% (8) obesidade grave. Quanto à circunferência da cintura 36\% (27) dos escolares apresentou risco para doenças cardiovasculares. Em relação ao percentual de gordura houve prevalência de classificação muito alta 46,7\% (35). Foi constatado que há correlação entre o consumo de ultraprocessados e uso de aparelhos eletrônicos assim como, entre o uso de aparelhos eletrônicos e o estado nutricional. Conclusão: Os alimentos ultraprocessados estão inseridos fortemente no consumo alimentar de escolares e a escolha destes alimentos, possui uma grande influência da mídia. A maioria das crianças pesquisadas se apresenta com excesso de peso, aumentando o risco de desenvolverem doenças crônicas não transmissíveis.

Palavras-chave: Mídia, Ultraprocessados, Alimentação de escolares.

\begin{abstract}
Objective: The aim of this study was to analyze the media's influence over consumption of ultra-processed foods and interference of nutritional status of children students. Methods: This study is descriptive and has a transversal approach. The researchers collected anthropometric data, consumption of ultra-processed foods and use of electronic devices. The research was carried out in a private school in the City of Teresina, capital of the Piauí State, Brazil. We selected 75 children students of morning and afternoon shifts, of both sexes, aged 7 to 10 years. Results: A higher prevalence of overweight was observed, being $22.7 \%$ (17) overweight, $14.7 \%$ (11) obesity and $10.7 \%$ (8) severe obesity. Regarding waist circumference, $36 \%$ (27) of children students presented a risk for cardiovascular diseases. Regarding fat percentage, there was a very high prevalence of $46.7 \%$ (35). There was a correlation between ultra-processed consumption and use of electronic devices as well as between the use of electronic devices and nutritional status. Conclusion: Ultra-processed foods are strongly inserted in the food consumption of children students and the choice of these foods has a great influence of the media. Most of the children surveyed are overweight, increasing the risk of developing chronic non-communicable diseases.
\end{abstract}

Key words: Media, Ultra-processed, Elementary students' nutrition.

${ }^{1}$ Centro Universitário Santo Agostinho - UNIFSA, Teresina-PI. *E-mail: jjanebarbosa03@gmail.com 


\section{RESUMEN}

Objetivo: Analizar la influencia de los médios de comunicación em el consumo de alimentos ultraprocesados por escolares y su relación con su estado nutricional. Métodos: Estúdio descriptivo, de enfoque transversal. Se recopilo, datos antropométricos, consumo de alimentos ultraprocesados y uso de aparatos electrónicos, La investigación se realizo en una escuela de La red privada em El municipio de Teresina-PI. Se seleccionaron, 75 escolares, de los turnos mañana y tarde, de ambos sexos, conedad de 7 a 10 años. Resultados: Hubo una mayor prevalencia de sobrepeso, con 22,7\% (17) de sobrepeso, obesidad de $14.7 \%$ (11) y obesidad severa $10,7 \%$ (8). En cuanto a cintura circunferencia $36 \%$ (27) del riesgo de enfermedades cardiovasculares presenta la escuela. En relación con el porcentaje de grasa hubo prevalencia de muy alta clasificación $46.7 \%$ (35). Se ha encontrado que existe correlación entre el consumo de ultraprocessados y uso de dispositivos electrónicos, así como entre el uso de dispositivos electrónicos y estado nutricional. Conclusión: los alimentos ultraprocessados se introducen en el consumo de alimentos de la escuela y la elección de estos alimentos tiene una gran influencia de los medios de comunicación. Mayoría de los niños encuestados presentada exceso de peso, aumenta el riesgo de desarrollar enfermedades crónicas no transmisibles.

Palabras clave: Medios, Ultraprocesados, Alimentación de escolares.

\section{INTRODUÇÃO}

A publicidade está presente em diversos momentos do nosso dia a dia, pois somos impactados continuamente, durante toda a vida, por anúncios de produtos e serviços que podem ser capazes de satisfazer nossas necessidades e mexer com os nossos desejos (ALMEIDA RG, 2016).

E através de apelos promocionais e propagandas de alimentos, por exemplo, a mídia influencia expressivamente o consumo alimentar infantil, por se tratar do público mais vulnerável, sendo um meio capaz de influenciá-los a hábitos e preferências que prosseguirão até a vida adulta (SANTANA M et al., 2015).

No caso do público infantil, as propagandas de produtos alimentícios têm como objetivo a formação de novos consumidores e, para isso, utiliza-se da mobilização de suas fantasias, forjando uma sensação de felicidade ao adquirir determinado produto, o que se torna fundamental no processo de convencimento dos pais, que são os responsáveis diretos pela compra (SPANIOL AM, 2014).

A publicidade voltada a esta faixa etária, conta com atrativos para seduzir este consumidor, dando o poder de persuasão e obtenção de reconhecimento ao universo infantil, por meio de associação com brinquedos, desenhos animados, personagens e da marca. Com isso, despertou-se nas empresas o interesse em produzir meios de consumo direcionados ao público infantil. Nos dias atuais, o tempo que as crianças gastam em frente à televisão, celular e computador vem crescendo absurdamente. Soma-se a isso o fato de que grande parte das propagandas apresentadas na televisão exerce influência negativa nas escolhas e hábitos em relação ao alimento pelas crianças (OLIVEIRA TS e POLL FA, 2017).

Assistir televisão, utilizar celular, tablet, dentre outros aparelhos eletrônicos, são atividades que demandam um baixo gasto energético, pois para realizá-las, as crianças permanecem apenas paradas por um longo período, não dando lugar a atividades vigorantes como correr, pular e etc, o que promove o sedentarismo, podendo ocasionar sobrepeso e/ou obesidade, além de poder interferir nas escolhas e hábitos alimentares das mesmas (MAS MD et al., 2018).

O padrão alimentar associado a problemas de saúde, como obesidade e doenças crônicas não transmissíveis (DCNT), como diabetes, hipertensão, dislipidemia, dentre outras, é caracterizado pelo baixo consumo de alimentos com fontes de vitaminas e fibras, ou seja, frutas, legumes e verduras, assim como pelo consumo excessivo de alimentos de alta densidade energética e ricos em gorduras, açúcares e sal, características dos alimentos processados e industrializados (BALDISSERA G et al., 2015). 
A alimentação cumpre um papel fundamental durante todo o ciclo de vida. Entre as diferentes fases da vida, pode-se destacar a criança em idade escolar, pois, nesse período, os hábitos alimentares adquiridos podem se perpetuar até a fase adulta (ALVES YF et al., 2018).Por esse motivo, o corpo necessita de aporte nutricional adequado para o crescimento e desenvolvimento completo e saudável nesta etapa (MIRANDA VPN et al, 2014).

Alimentos ultraprocessados são formulações da indústria, ricos em sódio, açúcar, gorduras e pobres em fibras, vitaminas e minerais, que têm se tornado cada vez mais acessíveis a todos os públicos, por serem mais palatáveis, atrativos e demandando pouco ou nenhum tempo de preparo. Com isso, estes alimentos possuem grande potencial para aumentar o risco de obesidade, diabetes, doenças cardiovasculares e de alguns tipos de câncer (BUENO MM et al., 2019).

Sendo assim, a alimentação dos escolares deve ser saudável, equilibrada e adequada em quantidade e qualidade de nutrientes, pois esta, é de suma importância para o desenvolvimento cognitivo, melhorando o aprendizado e auxiliando na prevenção de doenças crônicas não transmissíveis (MELLO AV et al., 2016).O presente trabalho teve como objetivo analisar a influência da mídia no consumo de alimentos ultraprocessados por escolares e sua relação com seu estado nutricional.

\section{MÉTODOS}

A pesquisa foi iniciada após a aprovação do Comitê de Ética em Pesquisa em Seres Humanos, sob o parecer 2.930.938 de 01/10/2018. A coleta de dados ocorreu no período de novembro de 2018 a março de 2019, por abordagem aos escolares matriculados na instituição. Os responsáveis legais e os participantes aceitaram assinar, respectivamente, o Termo de Consentimento Livre e Esclarecido (TCLE) e o Termo de Assentimento Livre e Esclarecido (TALE).

Trata-se de um estudo descritivo, de abordagem transversal e foi realizado em uma escola da rede privada no município de Teresina, no estado do Piauí. A população total é composta por 92 alunos, e a partir do cálculo estatístico foi determinada a amostra de 75 escolares, dos turnos manhã e tarde, de ambos os sexos, com idade de 7 a 10 anos, sendo garantida a participação apenas de alunos devidamente matriculados na instituição, mediante autorização dos pais e delas mesmas por meio da assinatura do TCLE e do TALE, respectivamente.

A coleta de dados foi realizada por meio de aplicação de um formulário sobre consumo alimentar e uso de aparelhos eletrônicos, elaborado pelas pesquisadoras, composto por questões que abordam a presença de mídia e do consumo de alimentos ultraprocessados no cotidiano dos escolares e também os dados pessoais e antropométricos. Para avaliação do estado nutricional dos escolares foi utilizado o Índice de Massa Corporal (IMC), gráfico Peso para Idade (P/I), Estatura para Idade (E/I) e IMC para idade (IMC/I) e para a classificação do diagnóstico foram utilizados os pontos de corte propostos pela Organização Mundial de Saúde.

As correlações entre consumo de alimentos processados e uso de parelhos eletrônicos, uso das mídias e estado nutricional, foram feitas utilizando o coeficiente $V$ de Cramer que é utilizado para medir a associação entre duas variáveis qualitativas com mais de 2 categorias. Os valores do teste variam de 0 a 1 . 0 valor 0 corresponde a ausência de associação entre as variáveis, valores próximos de zero correspondem a fraca associação e valores mais próximos de 1 correspondem a associação mais forte. $O$ valor de $p$ quando menor que 0,05 , significa associação estatisticamente significativa. Os dados foram tabulados no software Microsoft Office Excel e as análises foram realizadas no software Statistical Packages for the Social Sciences (SSP, versão 17.0) for Windows.

\section{RESULTADOS E DISCUSSÃO}

No período compreendido de Novembro de 2018 a Março de 2019, foram coletados dados de 75 escolares. Observou-se que ocorreu uma prevalência de peso adequado $69,3 \%(n=52)$, altura adequada $74,7 \%(n=56)$ e, em relação ao IMC, o excesso de peso apareceu em $48,1 \%(n=36)$ dos escolares dos quais, 22,7\% ( $n=17)$ apresentaram sobrepeso, $14,7 \%(n=11)$ obesidade e 10,7\% $(n=8)$ obesidade grave (Tabela 1). 
Tabela 1 - Distribuição dos escolares, segundo índices antropométricos.

\begin{tabular}{lll}
\hline Peso / Idade & $\mathbf{N}$ & $\%$ \\
\hline Peso adequado & 52 & 69,3 \\
Peso Elevado & 23 & 30,7 \\
\hline Estatura / Idade & & \\
\hline Altura adequada & 56 & 74,7 \\
Altura elevada & 19 & 25,3 \\
\hline IMC / Idade & & \\
\hline Magreza adequada & 4 & 5,3 \\
Adequado & 35 & 46,7 \\
Sobrepeso & 17 & 22,7 \\
Obesidade & 11 & 14,7 \\
Obesidade grave & 8 & 10,7 \\
\hline
\end{tabular}

Fonte: Dados da pesquisa, 2019.

Em uma pesquisa realizada por Perrone ACL et al. (2015), no parâmetro P/l, após a eutrofia, o estado nutricional mais prevalente foi o de baixo peso com o percentual de $7,48 \%$ para todas as crianças avaliadas e este resultado difere-se do encontrado na presente pesquisa, na qual não houve escolares com baixo peso, sendo após a eutrofia, o peso elevado o único parâmetro prevalente em $30,7 \%(n=23)$ dos escolares.

No parâmetro $E / l$, observou-se uma prevalência de estatura adequada em $74,7 \%(n=56)$, seguida por estatura elevada em 25,3\% ( $n=19)$ dos escolares. No trabalho de Borges CQ et al. (2018), que teve como objetivo avaliar o perfil antropométrico e a oferta dos lanches consumidos pelos escolares de uma cidade do Semiárido baiano, foi possível observar que,dentre os estudados, $49 \%$ encontravam-se com baixa estatura para a idade, enquanto $19 \%$ encontravam-se com elevada estatura para a idade e apenas $32 \%$ com estatura adequada.

Ao avaliar o estado nutricional, observa-se que $48,1 \%(n=36)$ dos escolares apresentam algum tipo de excesso de peso (sobrepeso, obesidade, obesidade grave). O estudo realizado por Marques MS et al. (2015), que avaliou crianças de 7 a 10 anos em uma unidade de Estratégia Saúde da Família (ESF), matriculadas nas redes de ensino pública e privada, observou maior prevalência de sobrepeso e obesidade em escolares provenientes da rede privada.

O excesso de peso é considerado como uma das principais ameaças à saúde no mundo, e na infância, sua presença mostra-se um preditor de risco para maior predomínio na vida adulta (COSTA MC et al., 2018).

Tabela 2 - Relação entre a circunferência da cintura e o risco de doenças cardiovasculares.

\begin{tabular}{ccccc}
\hline Risco de doenças cardiovasculares & N & $\%$ & Média & p-valor \\
\hline Sim & 27 & 36 & $70,3 \pm 9,7$ & \\
Não & 48 & 64 & $59,1 \pm 5,3$ & $<0,05^{\star *}$ \\
\end{tabular}

Fonte: Dados da pesquisa, 2019.

A Relação Cintura-Estatura (RCE) tem sido utilizada para avaliar a localização da gordura corporal, e para determinada estatura, há um grau aceitável para gordura armazenada na região abdominal sendo em crianças o valor de RCE 0,5 um indicativo de maior risco para doenças cardiovasculares em comparação com a $\mathrm{RCE}<0,5$. A maioria dos escolares não apresentou risco para doenças cardiovasculares, no entanto, $36 \%$ $(n=27)$ dos avaliados apresentaram circunferências elevadas, sendo a média da circunferência da cintura dos indivíduos que possuem risco de doenças cardiovasculares, estatisticamente, maior que a dos que não possuem risco (Tabela 2). 
Segundo Vieira SA et al. (2017), estudos epidemiológicos e clínicos têm mostrado que, a localização e a distribuição da gordura corporal, independentemente do excesso de peso, estão associadas a fatores de risco cardiometabólicos já em fases iniciais da vida, como a infância e a adolescência.

Com relação ao percentual de gordura corporal (\%GC), estimado pelo somatório das dobras tricipital e subescapular, o atual estudo identificou uma prevalência de classificação muito alta em 46,7\% ( $n=35)$, sendo

\begin{tabular}{|c|c|c|c|c|c|}
\hline Variáveis & Celular & Tablet & Computador & Videogames & Televisão \\
\hline Refrigerantes & $\begin{array}{c}0,151 \\
p=0,821\end{array}$ & $\begin{array}{c}0,234 \\
p=0,195\end{array}$ & $\begin{array}{c}0,126 \\
p=0,936\end{array}$ & $\begin{array}{c}0,222 \\
p=0,273\end{array}$ & $\begin{array}{c}0,288 \\
p=0,053\end{array}$ \\
\hline Hambúrguer & $\begin{array}{c}0,135 \\
p=0,906\end{array}$ & $\begin{array}{c}0,193 \\
p=0,500\end{array}$ & $\begin{array}{c}0,185 \\
p=0,563\end{array}$ & $\begin{array}{c}0,236 \\
p=0,187\end{array}$ & $\begin{array}{c}0,323 \\
p=0,016^{\star *}\end{array}$ \\
\hline Pizza & $\begin{array}{c}0,190 \\
p=0,521\end{array}$ & $\begin{array}{c}0,203 \\
p=0,410\end{array}$ & $\begin{array}{c}0,143 \\
p=0,867\end{array}$ & $\begin{array}{c}0,201 \\
p=0,431\end{array}$ & $\begin{array}{c}0,226 \\
p=0,226\end{array}$ \\
\hline Batata Frita & $\begin{array}{c}0,279 \\
p=0,041^{\star \star}\end{array}$ & $\begin{array}{c}0,161 \\
p=0,757\end{array}$ & $\begin{array}{c}0,206 \\
p=0,388\end{array}$ & $\begin{array}{c}0,296 \\
p=0,019^{\star \star}\end{array}$ & $\begin{array}{c}0,142 \\
p=0,807\end{array}$ \\
\hline Sorvete & $\begin{array}{c}0,164 \\
p=0,736\end{array}$ & $\begin{array}{c}0,133 \\
p=0,915\end{array}$ & $\begin{array}{c}0,197 \\
p=0,465\end{array}$ & $\begin{array}{c}0,280 \\
p=0,039^{\star \star}\end{array}$ & $\begin{array}{c}0,323 \\
p=0,016^{\star *}\end{array}$ \\
\hline $\begin{array}{l}\text { Biscoito } \\
\text { recheado }\end{array}$ & $\begin{array}{c}0,314 \\
p=0,008^{\star *}\end{array}$ & $\begin{array}{c}0,162 \\
p=0,750\end{array}$ & $\begin{array}{c}0,183 \\
p=0,579\end{array}$ & $\begin{array}{c}0,231 \\
p=0,211\end{array}$ & $\begin{array}{c}0,176 \\
p=0,589\end{array}$ \\
\hline Chocolate & $\begin{array}{c}0,211 \\
p=0,351\end{array}$ & $\begin{array}{c}0,286 \\
\mathrm{p}=0,030^{\star \star}\end{array}$ & $\begin{array}{c}0,197 \\
p=0,462\end{array}$ & $\begin{array}{c}0,285 \\
p=0,032^{\star \star}\end{array}$ & $\begin{array}{c}0,192 \\
p=0,478\end{array}$ \\
\hline $\begin{array}{l}\text { Sucos e } \\
\text { Achocolatado }\end{array}$ & $\begin{array}{c}0,184 \\
p=0,573\end{array}$ & $\begin{array}{c}0,281 \\
p=0,038^{\star *}\end{array}$ & $\begin{array}{c}0,190 \\
p=0,521\end{array}$ & $\begin{array}{c}0,171 \\
p=0,679\end{array}$ & $\begin{array}{c}0,144 \\
p=0,796\end{array}$ \\
\hline Petisco & $\begin{array}{c}0,257 \\
p=0,098\end{array}$ & $\begin{array}{c}0,224 \\
p=0,254\end{array}$ & $\begin{array}{c}0,204 \\
p=0,401\end{array}$ & $\begin{array}{c}0,268 \\
p=0,063\end{array}$ & $\begin{array}{c}0,248 \\
p=0,162\end{array}$ \\
\hline Bombons & $\begin{array}{c}0,177 \\
p=0,634\end{array}$ & $\begin{array}{c}0,218 \\
p=0,294\end{array}$ & $\begin{array}{c}0,214 \\
p=0,326\end{array}$ & $\begin{array}{c}0,115 \\
p=0,965\end{array}$ & $\begin{array}{c}0,253 \\
p=0,143\end{array}$ \\
\hline
\end{tabular}

este resultado preocupante, pois colabora para o desenvolvimento precoce de doenças crônicas (Tabela 3).

Quadro 1 - Correlação do uso de aparelhos eletrônicos e o consumo de alimentos ultraprocessados.

Fonte: Dados da pesquisa, 2019.

Tabela 3 - Distribuição dos escolares, de acordo com o percentual de gordura corporal.

\begin{tabular}{lcc}
\hline Classificação \%GC & $\mathbf{N}$ & $\%$ \\
\hline Ótimo & 12 & 16 \\
Moderadamente alto & 10 & 13,3 \\
Alto & 18 & 24 \\
Muito alto & 35 & 46,7 \\
\hline
\end{tabular}

Fonte: Dados da pesquisa, 2019.

Em um estudo realizado por Miranda JMC et al (2015), os resultados encontrados demonstram uma prevalência de sobrepeso, obesidade e de alto percentual de gordura corporal observado em escolares da rede privada de ensino. Além disso, o estudo evidenciou correlação positiva entre o IMC e o percentual de gordura corporal em crianças de ambos os sexos, a qual pode ser considerada como fator de risco para o desenvolvimento precoce de doenças crônicas.

Nesta pesquisa, foi aplicada uma tabela para verificar a frequência de consumo de alimentos ultraprocessados como refrigerantes, hambúrgueres, chocolate, sorvete e etc, e outa para a frequência de uso de aparelhos eletrônicos como celular, televisão, tablet, computador e vídeo game. E de acordo com os resultados obtidos foram verificadas correlações significativas entre ambas as variáveis. (Quadro 1).

Um estudo realizado por Pazin DC et al. (2017) demonstra a importância da medida da circunferência da cintura em crianças, pois sugere que a circunferência da cintura aumentada não está sempre associada com IMC elevado, particularmente quando o IMC estiver dentro da faixa de normalidade. 
Os resultados do Quadro 1 apontaram que existe associação significativa entre o consumo de hambúrgueres e a utilização de televisão. Também foi significativa a associação entre o consumo de batatas fritas com ouso do celular e de videogames. O consumo de sorvete foi associado significativamente com 0 uso de videogames e televisão. O biscoito recheado foi estatisticamente associado ao uso do celular.

A associação entre o consumo de chocolate e a utilização de tablets e videogames também apontou associação estatística. Por fim, o consumo de sucos e achocolatado também foram associados significativamente com a utilização de tablets.

Fidencio $\mathrm{J}$ et al. (2018) apontam que o sedentarismo favorece a obesidade e tem como principais hábitos o uso de televisores, celulares, vídeo games, tablets e computadores, que foram os substitutos das brincadeiras que demandavam um maior gasto energético. Além disso, em sua maioria, as crianças, quando estão de frente a uma tela, são expostas a grande parte das propagandas que oferecem alimentos não nutritivos e com alto teor calórico, sendo que $53 \%$ dessas propagandas são sobre refrigerantes e lanches prontos.

Uma pesquisa feita por Guse DEC et al. (2017), com alunos de uma Escola de Educação Básica na região noroeste do Rio Grande do Sul, que avaliou os lanches dos escolares nos turnos manhã e tarde, verificou um elevado consumo de alimentos processados, uma vez que $87,86 \%$ dos alunos relataram consumir esses alimentos.

O estudo de D’Ávila HF e Kirsten VR (2017) mostrou que a presença de alimentos ultraprocessados nas cantinas escolares e a publicidade relacionada a eles, são fatores que contribuem com as escolhas e preferências dos escolares por estes alimentos, os quais são mais agradáveis ao paladar e de baixo preço quando comparados a alimentos naturais, além de demandarem um consumo excessivo por não suprirem as necessidades energéticas nessa faixa etária.

A forte associação entre o uso da mídia e os hábitos alimentares se dá pelo fato das crianças serem um público mais vulnerável aos apelos da mídia, uma vez que elas ainda não possuem personalidade ou opinião formada, sendo estas facilmente influenciáveis. Assim, também se faz importante ressaltar, a associação significativa entre a mídia e o estado nutricional através do IMC em todas as suas variações sendo elas, Magreza, Adequado, Sobrepeso, Obesidade e Obesidade Grave as quais, mostraram correlação com o uso de tablet (Quadro 2).

Quadro 2- Correlação entre as mídias e a classificação do estado nutricional.

\begin{tabular}{|c|c|c|c|c|c|}
\hline Variáveis & Celular & Tablet & Computador & Videogame & Televisão \\
\hline & 0,226 & $\mathbf{0 , 4 2 7}$ & 0,242 & 0,235 & 0,206 \\
IMC & $\mathrm{p}=0,846$ & $\mathbf{p}=\mathbf{0 , 0 0 0 ^ { * * }}$ & $\mathrm{p}=0,357$ & $\mathrm{p}=0,412$ & $\mathrm{p}=0,605$ \\
\hline
\end{tabular}

Fonte: Dados da pesquisa, 2019.

O presente estudo mostra uma maior associação entre o uso do tablet e a classificação do estado nutricional das crianças avaliadas, em que a influência da utilização do tablet se sobrepõe ao da TV, demonstrando uma mudança em relação ao estudo realizado por Souza GR (2018), em que os pais confirmam que os filhos têm acesso às diversas opções, entre elas se destacam: televisão (36\%); celular/tablet (25\%) e internet (19\%). Quando relacionado à televisão, para $63 \%$ dos pais, as crianças passam entre 2 e 4 horas diárias com este meio de comunicação.

\section{CONCLUSÃO}

$\mathrm{Na}$ presente pesquisa, observou-se uma maior prevalência de excesso de peso, risco de doenças cardiovasculares associados à circunferência da cintura elevada, além de um predomínio de percentual de gordura muito alto. As correlações entre o consumo de ultraprocessados e uso de aparelhos eletrônicos, assim como do uso de mídias com o estado nutricional se apresentaram significativas. Diante disto, conclui-se que estes produtos alimentícios estão inseridos fortemente no consumo alimentar dos escolares e a escolha destes, possui uma 
grande influência da mídia, a qual se utiliza do lúdico para despertar o desejo e influenciar a compra, sendo o público infantil mais vulnerável por não possuírem personalidade ou opinião formada. Os resultados do estudo se mostram preocupantes, pois a maioria das crianças pesquisadas relatou consumir estes alimentos, e se apresenta com excesso de peso ou obesidade, aumentando o risco destas desenvolverem doenças crônicas não transmissíveis.

\section{REFERÊNCIAS}

1. ALMEIDA RG. Criança vê, criança imita: a publicidade e a construção dos hábitos alimentares. Monografia (Publicidade e Propaganda), Universidade Católica de Brasília, Brasília, 2016; 27f.

2. ALVES YF, et al. Teor de sódio e contribuição calórica de alimentos ultraprocessados no cardápio de uma unidade de alimentação e nutrição escolar em São Paulo-SP. Revista da Universidade Vale do Rio Verde, 2018; 16(1): 1-9.

3. BALDISSERA G, et al. Práticas e hábitos alimentares de crianças e adolescentes: a relação entre os aspectos socioculturais e midiáticos. Revista Caderno Pedagógico, 2015; 12(1): 289-300.

4. BORGES CQ, et al. Perfil antropométrico e o consumo alimentar de escolares em uma cidade do semiárido baiano. Revista Ciência (In) Cena, 2018; 1(7): 61-72.

5. BUENO MM, et al. Consumo de alimentos ultraprocessados por escolares de zona rural. Semina: Ciências Biológicas e da Saúde, 2019; 39 (2): 137-144.

6. COSTA MC, et al. Estado nutricional, práticas alimentares e conhecimentos em nutrição de escolares. Revista de Atenção à Saúde, 2018; 16(56): 12-17.

7. D'AVILA HF, KIRSTEN VR. Consumo energético proveniente de alimentos ultraprocessados por adolescentes. Rev Paul Pediatr, 2017; 35(1): 54-60.

8. FIDENCIO J, et al. Associação entre estado nutricional, horas de consumo de tela e de atividade física em adolescentes. RBONE-Revista Brasileira de Obesidade, Nutrição e Emagrecimento, 2018; 12 (72): 535-541.

9. GUSE DEC. Consumo de alimentos processados e ultraprocessados no lanche de escolares. Rio Grande do Sul, 2017.

10. MAS MD et al. Fatores alimentares e nutricionais associados ao hábito de assistir à televisão entre crianças de uma escola particular de Bento Gonçalves/RS. Revista Brasileira de Pesquisa em Saúde/BrazilianJournalof Health Research, 2018; 19(2): 36-45.

11. MARQUES MS, et al. Prevalência de sobrepeso e obesidade entre crianças de 7 a 10 anos atendidas em unidade de estratégia saúde da família - ESF. Revista Brasileira de Medicina de Família e Comunidade, 2015; 10(37): 1-9.

12. MELLO AV et al. Valor nutritivo de lanches consumidos por escolares de uma escola particular. Ciência\& Saúde, 2016; 9(2): 70-75.

13. MIRANDA JMC, et al. Prevalência de sobrepeso e obesidade infantil em instituições de ensino: públicas vs. privadas. RevBrasMed Esporte, 2015; 21(2): 104-107.

14. MIRANDA VPN et al. Estudo transversal sobre imagem corporal de adolescentes. Revista Psicologia: Teoria e Prática, 2014; 15(3): 151-162.

15. OLIVEIRA TS, POLL FA. A influência da publicidade na alimentação de escolares e sua relação com o excesso de peso. Cippus, 2017; 5(1): 29-38.

16. Pazin DC et al. Circunferência da Cintura está associada à Pressão Arterial em Crianças com Índice de Massa Corpórea Normal: Avaliação Transversal de 3417 Crianças Escolares. Cardiol, 2017; 109(6): 509-515.

17. PERRONE ACL, et al. Desenvolvimento infantil no interior do amazonas: avaliação antropométrica de escolares de 9 anos. Saúde \& Transformação Social/Health \& Social Change, 2015; 6(3): 42-49.

18. SANTANA M, et al. Influência da publicidade de alimentos direcionados ao público infantil na formação de hábitos alimentares. Rev. UNI-RN, 2015; 14(1/2): 125-136.

19. SPANIOL AM. Influência da publicidade de alimentos sobre a escolha alimentar de crianças e adolescentes de escolas públicas do Distrito Federal. Dissertação (Mestrado em Nutrição Humana) —Universidade de Brasília, Brasília, 2014; 168 .

20. SOUZA RG Consumo Infantil: A Influência da Mídia no Desejo de Compra das Crianças. Repositório de Trabalhos de Conclusão de Curso e Monografias, 2018.

21. VIEIRA SA, et al. Índice relação cintura-estatura para predição do excesso de peso em crianças. Revista Paulista de Pediatria, 2018; 36(1): 52-58. 